\title{
MST1 coordinately regulates autophagy and apoptosis in diabetic cardiomyopathy in mice
}

\author{
Mingming Zhang ${ }^{1,2} \cdot$ Lei Zhang $^{3} \cdot$ Jianqiang Hu ${ }^{1,2} \cdot$ Jie Lin $^{1,2} \cdot$ Tingting Wang ${ }^{1,2}$. \\ Yu Duan ${ }^{1,2}$ - Wanrong Man ${ }^{1,2} \cdot$ Jiaxu Feng ${ }^{1,2}$ - Lei Sun ${ }^{1,2} \cdot$ Hongbing $\mathrm{Jia}^{4}$ • Congye $\mathrm{Li}^{2}$. \\ Rongqing Zhang ${ }^{2}$ • Haichang Wang ${ }^{1,2}$ • Dongdong Sun ${ }^{1,2}$
}

Received: 3 April 2016 / Accepted: 14 July 2016 /Published online: 10 August 2016

(C) Springer-Verlag Berlin Heidelberg 2016

\begin{abstract}
Aims/hypothesis Diabetic cardiomyopathy (DCM) is associated with suppressed autophagy and augmented apoptosis in the heart although the interplay between the two remains elusive. The ability of mammalian sterile 20-like kinase 1 to regulate both autophagy and apoptosis prompted us to investigate it as a possible candidate in the progression of DCM.

Methods Wild-type, Mst1 (also known as Stk4) transgenic and Mst1-knockout mice were challenged with streptozotocin to induce experimental diabetes. In addition, cultured neonatal mouse cardiomyocytes were subjected to simulated diabetes to probe mechanisms.

Results Mst 1 knockout alleviated while Mst 1 overexpression aggravated cardiac dysfunction in diabetes. Diabetic Mst 1 transgenic mice exhibited decreased LC3 expression and
\end{abstract}

Mingming Zhang, Lei Zhang, Jianqiang $\mathrm{Hu}$ and Jie Lin contributed equally to this work.

Electronic supplementary material The online version of this article (doi:10.1007/s00125-016-4070-9) contains peer-reviewed but unedited supplementary material, which is available to authorised users.

Haichang Wang

wanghc@fmmu.edu.cn

Dongdong Sun

wintersun3@gmail.com

1 Department of Cardiology, Tangdu Hospital, Fourth Military Medical University, 1 Xinsi Road, Xi'an, Shaanxi 710038, People's Republic of China

2 Department of Cardiology, Xijing Hospital, Fourth Military Medical University, Xi'an, People's Republic of China

3 Department of Neurosurgery, Xijing Hospital, Fourth Military Medical University, Xi'an, People's Republic of China

4 Department of Cadre's Ward, PLA 323 Hospital, Xi'an, People's Republic of China enhanced protein aggregation. In contrast, typical autophagosomes were observed in diabetic Mst1-knockout mice with increased LC3 expression and reduced protein aggregation. Mst1 downregulation promoted autophagic flux as demonstrated by increased LC3-II and decreased p62 expression in the presence of bafilomycin A1. Furthermore, Mst1 overexpression increased, while Mst1 knockout decreased, cardiomyocyte apoptosis both in vivo and in vitro. Coimmunoprecipitation assays showed that Mst 1 overexpression promoted Beclin1 binding to B cell lymphoma 2 (Bcl-2) and induced dissociation of Bcl-2 from Bax in diabetic mice. Conversely, Mst1 knockout disrupted the Beclin1-Bcl-2 complex and enhanced the interaction between Bcl-2 and Bax. Conclusions/interpretation Mst1 knockout restores autophagy and protects against apoptosis in cardiomyocytes, en route to the rescue against DCM.

Keywords Autophagy $\cdot$ Beclin $1 \cdot$ Diabetes $\cdot$ MST1

$\begin{array}{ll}\text { Abbreviations } \\ \text { 3-MA } & \text { 3-Methyladenine } \\ \text { AMPK } & \text { Adenine mononucleotide protein kinase } \\ \text { Bcl-2 } & \text { B cell lymphoma } 2 \\ \text { BH3 } & \text { Bcl-2 homology } 3 \\ \text { DCM } & \text { Diabetic cardiomyopathy } \\ \text { DM } & \text { Diabetic mouse group } \\ { }^{18} \text { F-FDG } & {\left[{ }^{18} \text { F]fluorodeoxyglucose }\right.} \\ \text { GFP } & \text { Green fluorescent protein } \\ \text { LVEF } & \text { Left ventricular ejection fraction } \\ \text { LVFS } & \text { Left ventricular fraction shortening } \\ \text { LVESD } & \text { Left ventricular end-systolic diameter } \\ \text { LVEDD } & \text { Left ventricular end-diastolic diameter } \\ \text { MST1 } & \text { Mammalian sterile 20-like kinase 1 } \\ \text { NTg } & \text { Non-transgenic mouse group }\end{array}$




$\begin{array}{ll}\text { PET/CT } & \begin{array}{l}\text { Positron emission tomography-computed } \\ \text { tomography }\end{array} \\ \text { PI3K } & \begin{array}{l}\text { Phosphoinositide 3-kinase } \\ \text { STZ }\end{array} \\ \text { Streptozotocin }\end{array}$

\section{Introduction}

Diabetes mellitus is projected to reach pandemic proportions over the next few decades, with a WHO-estimated global prevalence of 330 million in 2025 [1]. Diabetic cardiomyopathy (DCM) is a unique heart muscle-specific disease that increases the risk of heart failure and mortality in diabetic patients independent of coronary artery disease or hypertension. Two-thirds of diabetic patients die from cardiovascular disease, highlighting the pertinent need for understanding and management of cardiovascular complications of diabetes including DCM [2-5].

Autophagy is a conservative bulk degradation mechanism through which cytosolic proteins and organelles are sequestrated into autophagosomes to be degraded by lysosomes. There is ample evidence for the essential role of autophagy in maintaining organelle function and protein quality via the removal of damaged organelles and protein aggregates [6]. Accumulating findings have demonstrated that autophagy is centrally involved in DCM, where apparent metabolic disorders and impairment of cardiac function exist concurrently [7, 8]. Recent work from our group also showed inhibited cardiomyocyte autophagy in a mouse model of type 1 diabetes, suggesting that inhibition of autophagy may contribute to the pathogenesis of DCM [9].

Mammalian sterile 20-like kinase 1 (MST1) is a 487amino-acid serine-threonine kinase and forms part of the 'Hippo' signalling pathway, which regulates cell proliferation and organ size $[10,11]$. MST1 has been shown to serve as a key component of a multistep signalling pathway that controls the expression of genes associated with autophagy and apoptosis [6, 10-12]. More recent studies have suggested a cardinal role for MST1 in the development of cardiac dysfunction through inhibition of autophagy and facilitated accumulation of protein aggresomes [6]. Overexpression of MST1 initiates apoptosis, whereas inhibition of endogenous MST1 prevents apoptosis and cardiomyopathy in response to pathologically relevant stresses [11, 12]. Interestingly, our recent studies have found suppressed autophagy and augmented apoptosis in diabetic hearts. To this end, the current study was designed to investigate the potential role of MST1 in mediating diabetic cardiac injury and to elucidate the mechanism by which MST1 regulates autophagy and apoptosis in the pathogenesis of DCM.

\section{Methods}

Materials For details regarding materials, please refer to the electronic supplementary material (ESM) Methods.

Genetically modified mice $M s t 1$ (also known as $S t k 4$ )-knockout $\left(M s t 1^{-/}\right)$and $M s t 1$-transgenic (Tg-Mst 1$)$ mice were purchased from K\&D gene technology (WuHan, China) (C57BL/6 background). Western blotting and real-time PCR were used to screen $M s t 1^{-1-}$ and Tg-Mst1 mice. Age-matched male mice (6-8 weeks, 20-25 g) were used in all animal experiments. The experimenters were blind to group assignment and outcome assessment.

Experimental diabetes model Diabetes was induced in mice by i.p. injection of streptozotocin (STZ) $(50 \mathrm{mg} / \mathrm{kg}$; dissolved in $0.1 \mathrm{~mol} / 1$ citrate buffer, $\mathrm{pH} 4.5$ ) daily for 5 days; mice were then maintained for another 3 months. Mice with glucose levels $\geq 16.6 \mathrm{mmol} / 1$ were classified as having diabetes. The mice were randomly divided into the following groups: (1) wild-type (WT); (2) Mst $1^{-/-}$; (3) diabetes (DM); (4) DM + $M s t 1^{-/-}$; (5) non-transgenic mice (NTg); (6) Tg-Mst1; (7) $\mathrm{DM}+\mathrm{NTg}$; (8) DM + Tg-Mst1. The diabetic mice were randomly assigned to receive treatment with or without AMPK activator metformin ( $200 \mathrm{mg} / \mathrm{kg}$ per day in drinking water) for 2 weeks. For autophagy inhibition, mice were injected intraperitoneally with autophagy inhibitor 3-methyladenine (3-MA) (10 mg/kg per day) for ten consecutive days.

$\left[{ }^{18} \mathrm{~F}\right]$ fluorodeoxyglucose positron emission tomographycomputed tomography to assess cardiac glucose metabolism Whole-body $\left[{ }^{18} \mathrm{~F}\right]$ fluorodeoxyglucose $\left({ }^{18} \mathrm{~F}-\mathrm{FDG}\right)$ positron emission tomography-computed tomography (PET/CT) scanning was performed using an animal PET/CT scanner (Mediso Nano PET/CT, Boston, MA, USA) as described previously [13]. See ESM Methods for details.

Echocardiography Echocardiography was conducted in M-mode using an echocardiography system with a $15 \mathrm{MHz}$ linear transducer (Vevo 2100; VisualSonics, Toronto, ON, Canada) as described previously [13]. See ESM Methods for details.

Immunoblotting The following antibodies were used: p-MST1(Thr183), MST1, Beclin1, p62, cleaved caspase-3, cleaved caspase-9, Bax, Bcl-2, LC3A/B, Atg7, AMPK, p-AMPK (Thr172), $\beta$-actin, GAPDH; and secondary antibodies (anti-mouse/rabbit IgG) conjugated with horseradish peroxidase (1:5000, Cell Signaling). See ESM Methods for details of the immunoblotting procedure and antibodies.

Primary neonatal mouse ventricular cardiomyocytes culture and transfection Primary cultures of ventricular 
cardiomyocytes were prepared from 1-day-old non-transgenic mice. Cells were cultured in DMEM supplemented with $10 \%$ fetal bovine serum (Hyclone SV30087.02, Logan, UT, USA) and $1 \%$ penicillin/streptomycin and maintained at $37^{\circ} \mathrm{C}$ in $5 \%$ $\mathrm{CO}_{2}$ as previously described [14].

Transduction of adenoviruses The adenovirus harbouring green fluorescent protein-LC3 (GFP-LC3) was purchased from GeneChem technology (Shanghai, China). The adenoviruses harbouring Mst1 (Ad-Mst1) (MOI:100), Mst1 shRNA (Ad-shMst1) (MOI:100) and control vectors for Ad-Mst1 (Ad-Control) and Mst1 shRNA (Ad-LacZ) were purchased from Hanbio Technology (Shanghai, China) and were transduced $24 \mathrm{~h}$ after transduction of GFP-LC3 (14). After 36 h, the cardiomyocytes were treated with either regular glucose medium $(5.5 \mathrm{mmol} / \mathrm{l})$ or high-glucose medium ( $30 \mathrm{mmol} / \mathrm{l})$ for $48 \mathrm{~h}$.

Detection of GFP-LC3, aggresomes and p62 Fluorescence microscopic detection of GFP-LC3, aggresomes and p62 were conducted according to the manufacturer's instructions as previously described [14]. See ESM Methods for details.

\section{Construction of short hairpin RNA adenoviral expression} vectors The generation of adenoviruses GFP-LC3 is described elsewhere [14].

Determination of cardiomyocyte apoptosis Cardiomyocyte apoptosis was determined by TUNEL staining, flow cytometric analysis and western blot evaluation as previously described [15]. See ESM Methods for details.

In vivo glucose homeostasis An IPGTT was performed to measure blood glucose levels. See ESM Methods for details.

Electron microscopy Autophagosomes and mitochondrial were detected by transmission electron microscopy. See ESM Methods for details.

Citrate synthase, chain complex activity and ATP content Citrate synthase, electron transport chain complex activity (complex I, II, III, IV and V) and the ATP content of the myocardium were measured according to the standard protocols. See ESM Methods for details.

Measurement of mitochondrial membrane potential JC-1 (Beyotime, Jiangsu, China) was employed to measure the mitochondrial membrane potential $(\Delta \Psi \mathrm{m})$ of cardiomyocytes. See ESM Methods for details.

Statistics Statistical analyses of differences between groups were carried out using unpaired Student's $t$ test, one-way ANOVA followed by a Fisher's post hoc comparison test or a two-way ANOVA with multiple post hoc comparisons. A $p$ value of less than 0.05 was considered significant. Statistical analyses were done using GraphPad Prism 7 (GraphPad Software, La Jolla, CA, USA).
Fig. 1 Mstl knockout preserves cardiac function in diabetic mice. (a) Representative images of PET/CT whole-body scans showing transverse, coronal and sagittal view. A higher intensity of red colour indicates a higher glucose level. (b) Quantification of accumulated ${ }^{18} \mathrm{~F}-\mathrm{FDG}$ in the heart. Relative accumulation of the radioactivity in particular regions of interest was expressed as identification $\%$ per gram (ID\%/g). (c-f) Measurements of LVEF, LVFS, LVESD and LVEDD. (g) Quantification of the early mitral diastolic wave/late mitral diastolic wave ratio (E/A ratio). Data are means and SEM $(n=12) . * p<0.05$ vs WT; ${ }^{\dagger} p<0.05$ vs Mst $^{-1-} ;{ }^{\star} p<0.05$ vs DM a

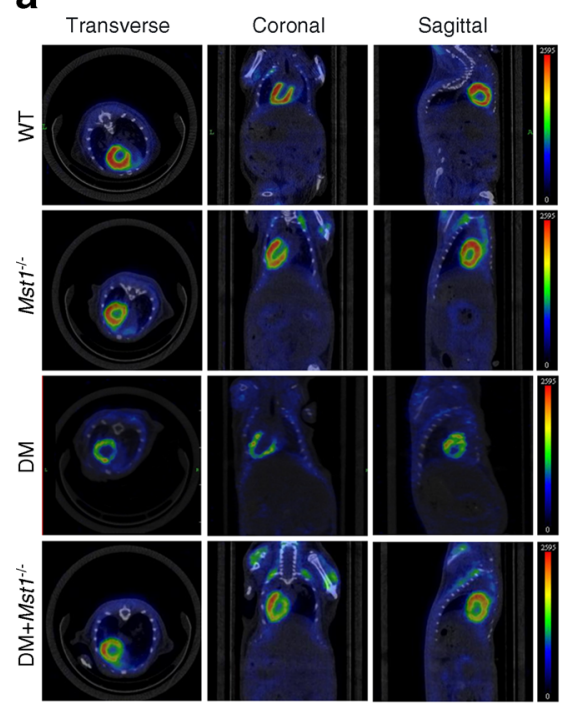

b

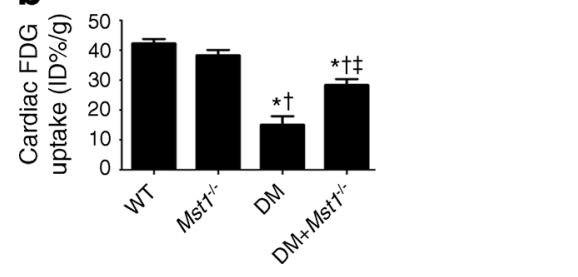

C

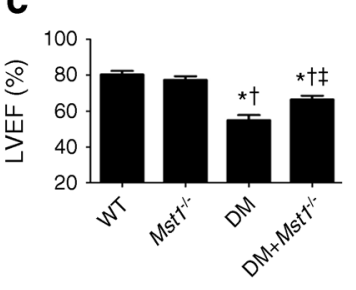

d

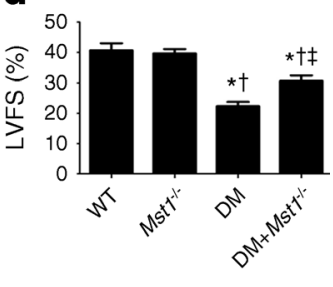

e

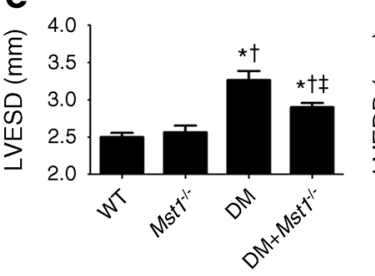

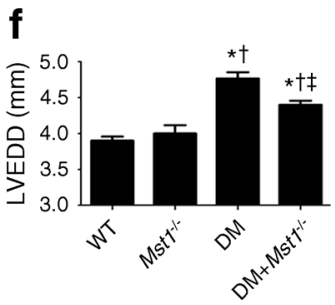

g

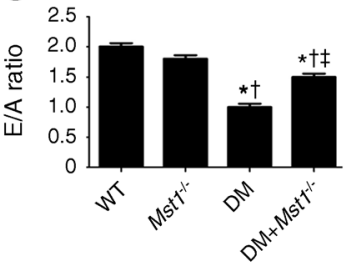




\section{Results}

Mst1 knockout alleviates cardiac dysfunction in diabetic mice ${ }^{18} \mathrm{~F}$-FDG imaging was used to quantify cardiac glucose metabolism. Diabetes led to a defective ${ }^{18} \mathrm{~F}-\mathrm{FDG}$ uptake in the heart and this defect was significantly improved by Mst 1 knockout (Fig. 1a, b). Representative M-mode echocardiograms are shown in ESM Fig. 1a. Left ventricular ejection fraction (LVEF) and left ventricular fraction shortening (LVFS) were significantly higher in the $\mathrm{DM}+M s t 1^{-/-}$group compared with the DM group (Fig. 1c, d). Mst1 knockout significantly inhibited the increase in left ventricular endsystolic diameter (LVESD) and left ventricular end-diastolic diameter (LVEDD) caused by diabetes (Fig. 1e, f). Representative mitral flow patterns from pulsed Doppler are shown in ESM Fig. 1b. Mst 1 knockout also improved diastolic function in diabetes as demonstrated by enhanced E/A ratio (Fig. 1g). Baseline variables of the mice are shown in ESM Table 1. The IPGTT showed that the mouse model of diabetes was constructed successfully (ESM Fig. 2).
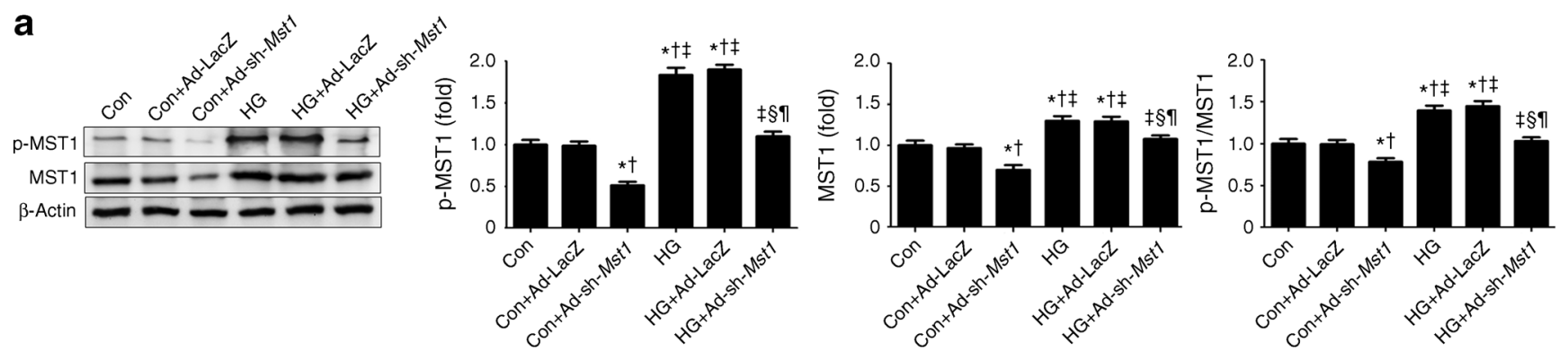

b

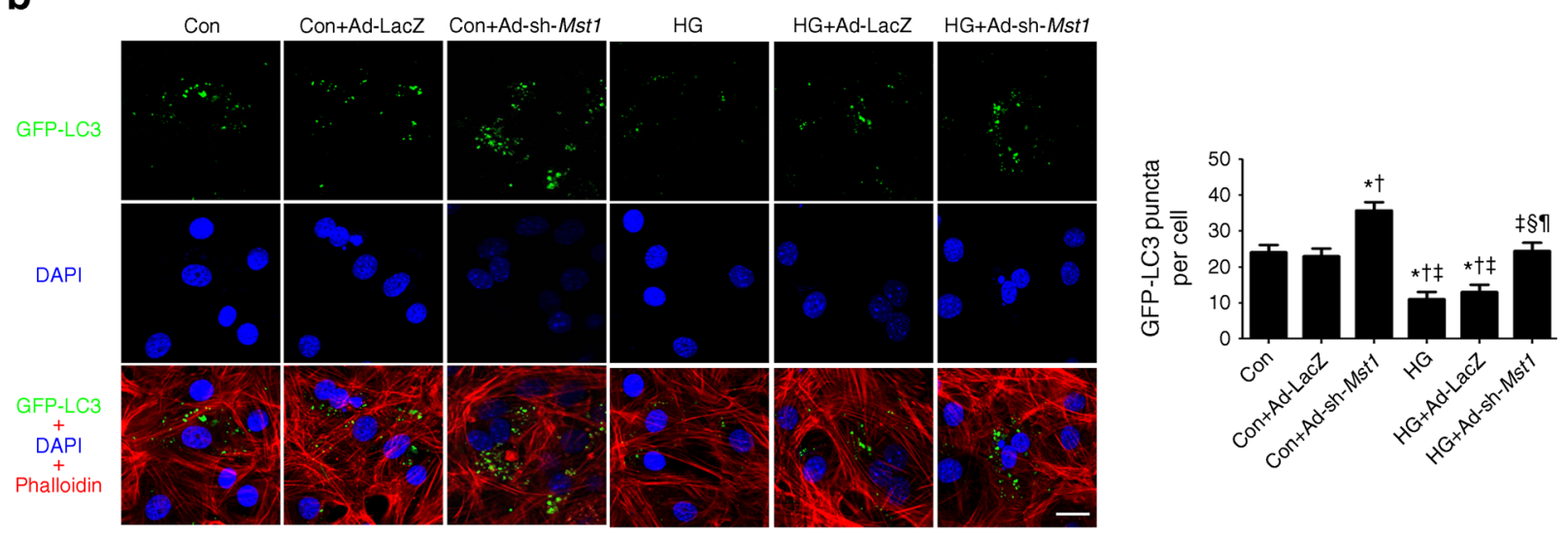

C

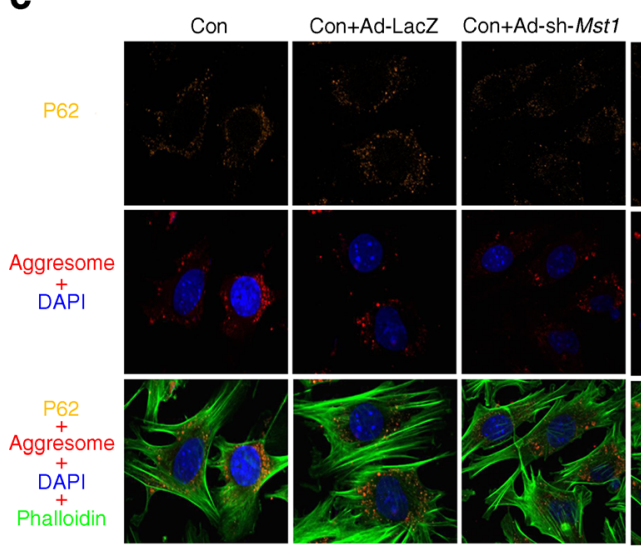

Fig. 2 Mst 1 knockout induces autophagy and reduces the accumulation of protein aggresomes and p62 in cardiomyocytes. (a) Representative blots and analysis of p-MST1 and MST1 expression. (b) Representative images and quantitative analysis of GFP-LC3 puncta. (c) Immunofluorescence staining for p62 (orange), DAPI (blue) and ProteoStat aggresome detection reagent (red). The number of
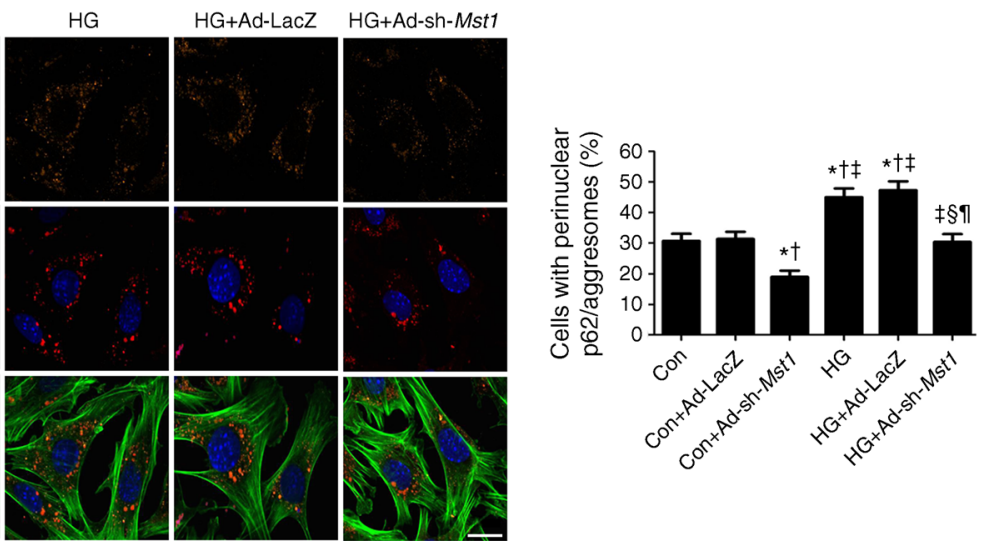

cardiomyocytes with perinuclear co-localisation of p62 and aggresomes (p62/aggresomes), indicated by yellow colour in the merged images, was counted ( $n=50$ in each group). ${ }^{*} p<0.05$ vs Con; ${ }^{\dagger} p<0.05$ vs Con + AdLacZ; ${ }^{\star} p<0.05$ vs Con + Ad-sh-Mst $1 .{ }^{\S} p<0.05$ vs HG; ${ }^{9} p<0.05$ vs HG + Ad-LacZ 
Mst1 knockout induces autophagy and reduces accumulation of protein aggresomes and p62 in cardiomyocytes There was a significant increase in p-MST1, MST1 and p-MST1/MST1 ratio after high-glucose culture and a significant reduction after Ad-sh-Mst1 transfection as evaluated by western blotting (Fig. 2a). In cardiomyocytes transduced with GFP-LC3, Ad-sh-Mst1 transfection increased green puncta number as compared with the control group under normal or high-glucose condition (Fig. 2b). In cardiomyocytes cultured with high-glucose medium, Ad-sh-Mst1 transfection significantly decreased accumulation of aggresomes and p62 (Fig. 2c).

Mst1 knockout induces autophagy by dissociation of Beclin1 and Bcl-2 The enhanced autophagic flux exerted by Ad-sh-Mst1 transfection was demonstrated by increased LC3-
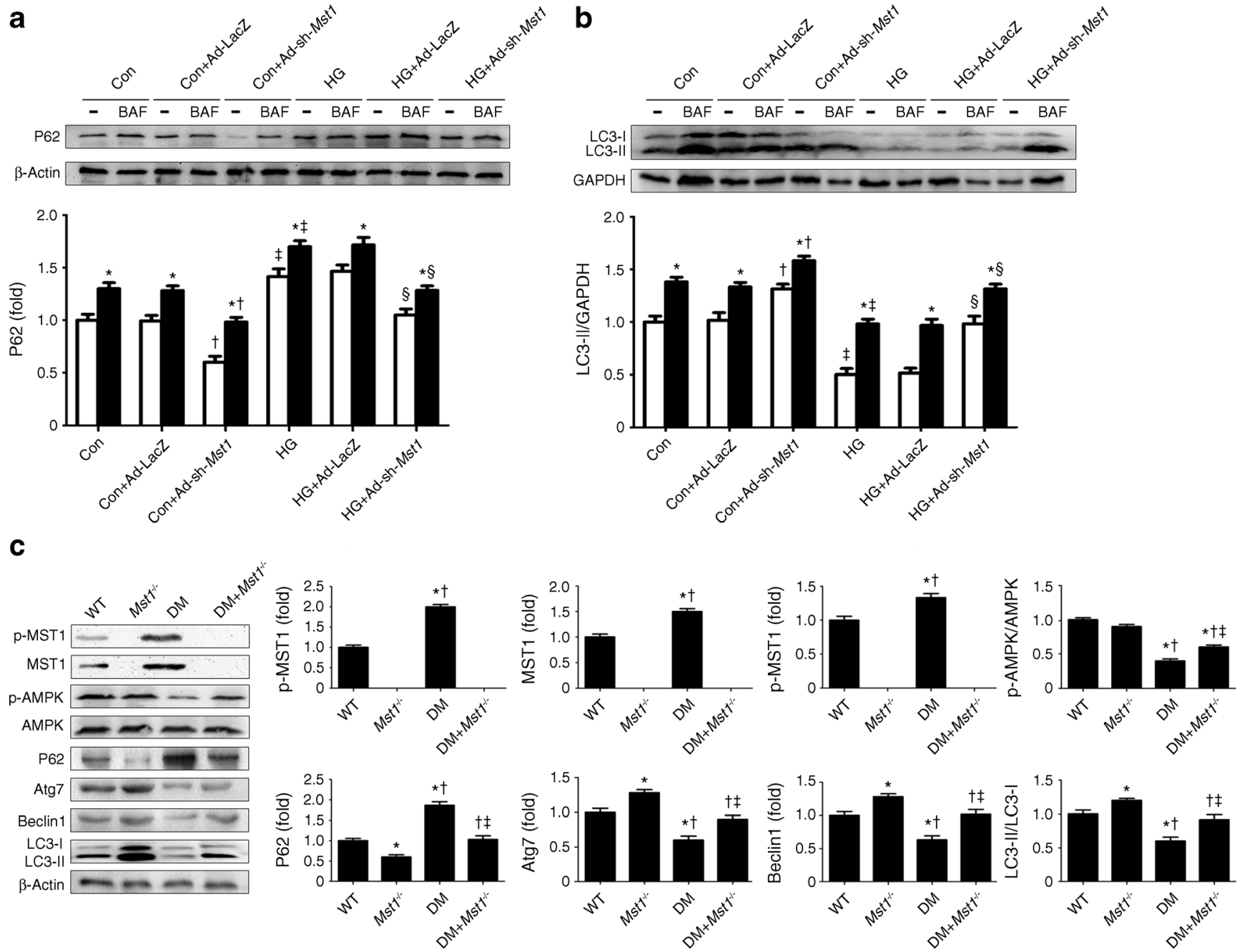
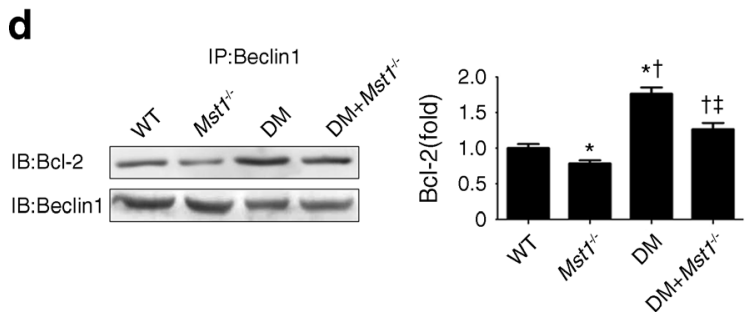

Fig. 3 Mst 1 knockout induces autophagy by regulating the association of Beclin1 and Bcl-2. (a, b) Representative blots and analysis of p62 (a) and LC3 (b) in the absence or presence of bafilomycin A1 (BAF). Black bars, BAF; white bars, BAF-free; * $p<0.05$ vs BAF-free treatment group; ${ }^{\dagger} p<0.05$ vs Con + Ad-LacZ group; ${ }^{\star} p<0.05$ vs Con group; ${ }^{\S} p<0.05$ vs HG + Ad-LacZ group. (c) Immunoblots and quantitative analyses of pMST1, MST1, p-AMPK, AMPK, p62, Atg7, Beclin1 and LC3-II/LC3-I.

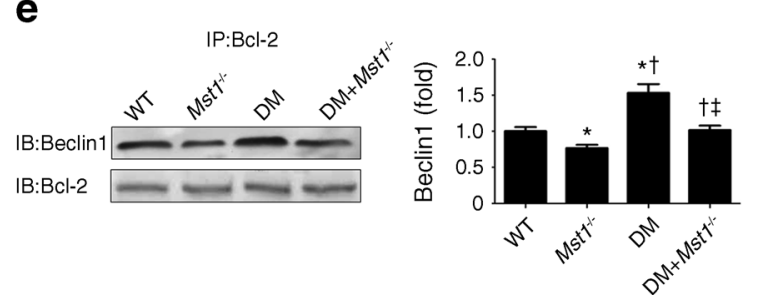

$* p<0.05$ vs WT; ${ }^{\dagger} p<0.05$ vs $M s t 1^{-1} ;{ }^{\dagger} p<0.05$ vs DM. (d, e) Beclin1 or Bcl-2 was immunoprecipitated (IP) from cell lysates, and Bcl-2 (d) or Beclin1 (e) in the immunoprecipitates was detected by western blot (IB). ${ }^{*} p<0.05$ vs WT; ${ }^{\dagger} p<0.05$ vs $M s t 1^{-1-} ;{ }^{\star} p<0.05$ vs DM. Data are means and $\operatorname{SEM}(n=12)$. Scale bars, $20 \mu \mathrm{m}$; BAF, bafilomycin A1; Con, control; $\mathrm{HG}$, high glucose 
a

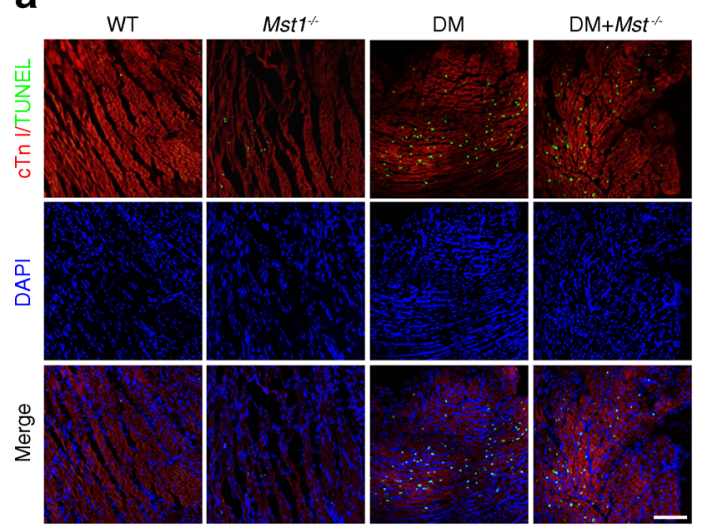

C
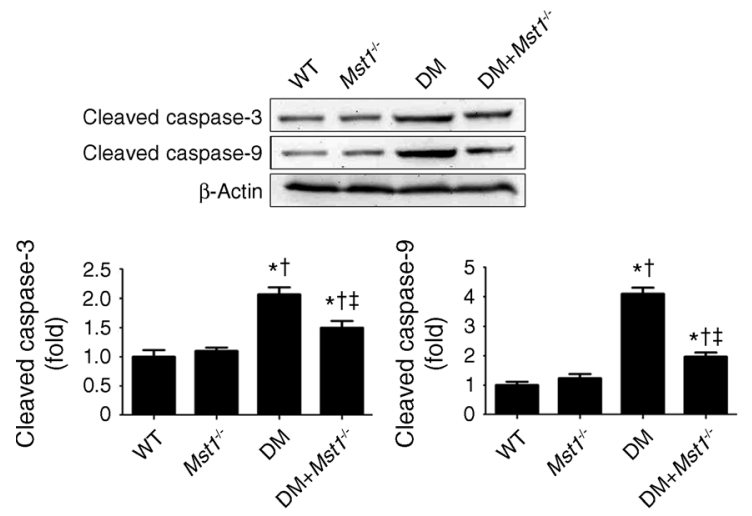

e

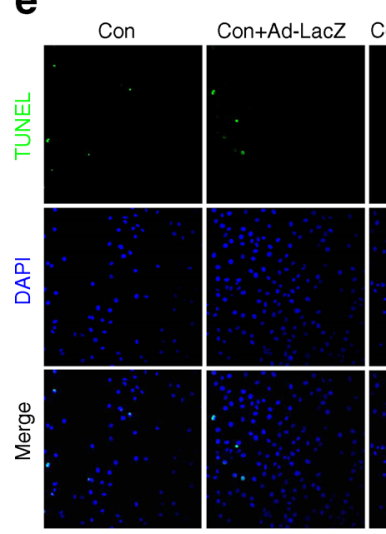

g
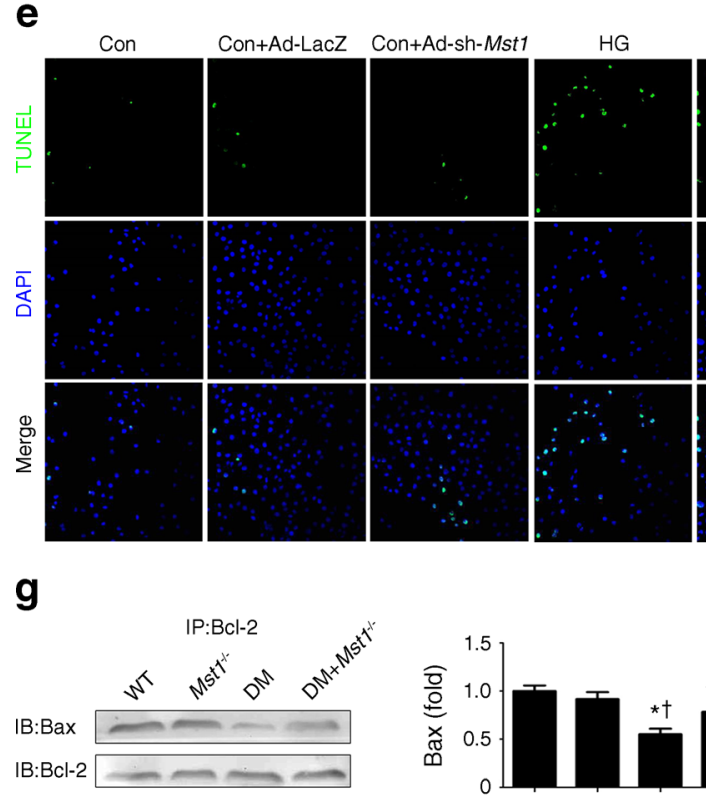

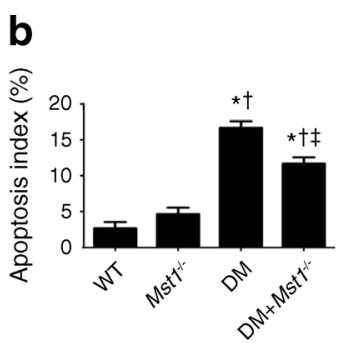

\section{d}

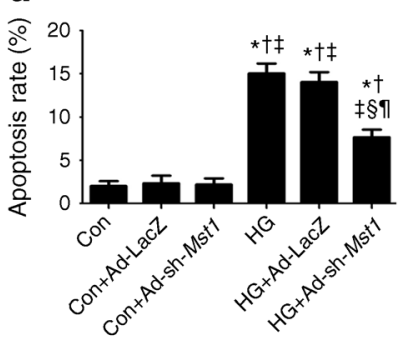

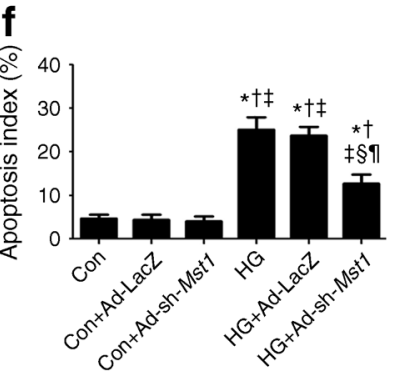

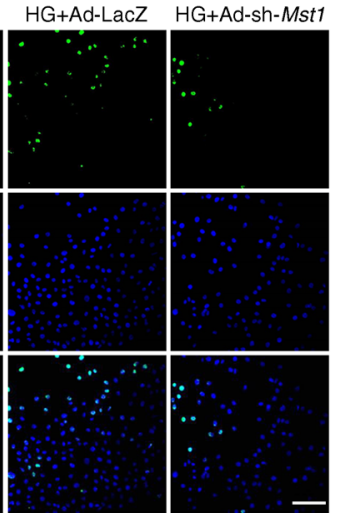

h
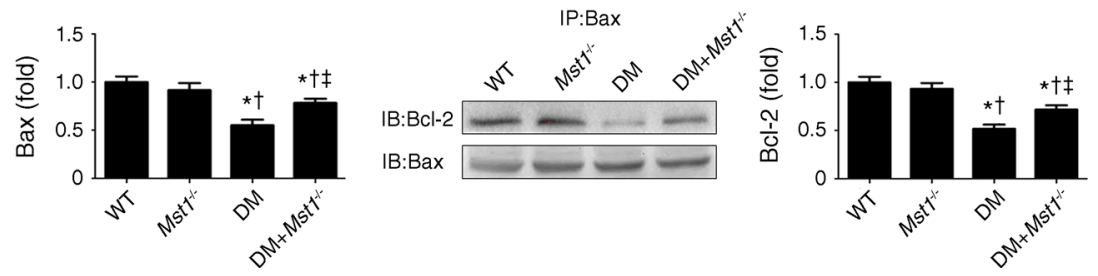

II/GAPDH ratio and decreased p62 expression in the presence of bafilomycin A1, a lysosomal inhibitor used to evaluate autophagic flux (Fig. 3a, b). In addition, there was a significantly elevated LC3-II/LC3-I ratio and a significantly lower level of p62 expression in the $M s t 1^{-/}$group as compared with

the WT group (Fig. 3c). The p-MST1/MST1 ratio was elevated, while Beclin1 expression and p-AMPK/AMPK ratio was decreased in diabetic hearts (Fig. 3c). Interestingly, the $\mathrm{p}$-AMPK/AMPK ratio was increased in the $\mathrm{DM}+M s t 1^{-/-}$ group compared with the DM group (Fig. 3c). 
Fig. 4 Mst1 knockout regulates the association of Bax and Bcl-2 and reduces the number of apoptotic cells. (a, b) Representative immunofluorescent images of staining with TUNEL (green), DAPI (blue) and Troponin T antibody (cTn I; red) (a) and quantitative analysis of apoptosis (b). Apoptosis index is the percentage of TUNELpositive myocytes over total nuclei determined by DAPI staining ( $n=50$ fields per group). (c) Representative gel blots and quantification of protein expression of cleaved caspase-3, cleaved caspase- 9 and $\beta$-actin (loading control). Data are means and SEM $(n=12) . * p<0.05$ vs WT; ${ }^{\dagger} p<0.05$ vs $M s t 1^{-1} ;{ }^{\ddagger} p<0.05$ vs DM. (d) Apoptosis rate = (late apoptotic cells + early apoptotic cells)/total cells $\times 100$. (e, f) Representative images of TUNEL-stained primary neonatal cardiomyocytes (e) and apoptotic index (f) ( $n=50$ fields per group). (g, h) Bax or Bcl-2 was immunoprecipitated (IP) from cell lysates, and $\mathrm{Bcl}-2$ or $\mathrm{Bax}$ in the immunoprecipitates was detected by western blot (IB). Data are means and SEM $(n=12)$. ${ }^{*} p<0.05$ vs Con; ${ }^{\dagger} p<0.05$ vs Con + Ad-LacZ; ${ }^{\star} p<0.05$ vs Con + Ad-sh-Mst $1 ;{ }^{\S} p<0.05$ vs HG; ${ }^{\pi} p<0.05$ vs HG + Ad-LacZ. Scale bars, $50 \mu \mathrm{m}$. Con, control, HG, high glucose

Co-immunoprecipitation assays and western blot analysis were performed to elucidate how MST1 inhibits autophagy. STZ-induced experimental diabetes promoted the interaction between Beclin1 and B cell lymphoma 2 (Bcl-2) (Fig. 3d, e). Mst1 knockout decreased the p-MST1/MST1 ratio, increased Beclin1 expression and consequently disrupted the association between Beclin1 and Bcl-2, suggesting that MST1 is a physiological kinase mediating the interaction between Beclin1 and Bcl-2 (Fig. 3c-e). Assessment of cardiac function revealed that 3-MA significantly depressed LVEF as well as LVFS and increased LVESD and LVEDD in diabetic mice.
Meanwhile, the protective effects of Mstl knockout were reversed by 3-MA administration in diabetic mice (ESM Fig. 3a-d). Autophagosomes, evaluated using transmission electron microscopy, were significantly increased in the $M s t 1^{-/}$group compared with the WT group regardless of diabetic state (ESM Fig. 4a). Ad-sh-Mst1 transfection significantly increased the number of autophagosomes as compared with the control group under normal and high-glucose conditions (ESM Fig. 4b). Furthermore, Mst 1 knockout improved mitochondrial biogenesis and mitochondrial function in diabetic mice (ESM Fig. 4c-f).

Mst1 knockout inhibits cardiomyocyte apoptosis by association of Bax and Bcl-2 Photomicrograph images showed that TUNEL-positive cardiomyocytes were more abundant in the DM group than in the WT group; this effect was negated by Mst 1 knockout (Fig. 4a, b). Concomitantly, levels of cleaved caspase-3 and cleaved caspase- 9 were downregulated by Mst 1 knockout in the setting of diabetes (Fig. 4c). Mst 1 knockout inhibited highglucose-induced cardiomyocyte apoptosis (Fig. 4d-f). Mst 1 knockout increased the association of Bax and Bcl-2 in diabetic mice, as shown by co-immunoprecipitation assays (Fig. 4g, h). The results of flow cytometry analysis showed that Ad-sh-Mst 1 transfection significantly decreased the apoptosis rate of cardiomyocytes as compared with the control group under highglucose conditions (ESM Fig. 5).
Fig. 5 Mst 1 overexpression aggravates cardiac dysfunction in diabetic mice. (a) Representative images of PET/CT whole-body scans. (b) Quantification of accumulated ${ }^{18} \mathrm{~F}$-FDG in the heart. Relative accumulation of the radioactivity in particular regions of interest was expressed as identification $\%$ per gram (ID\%/g). (c-f) Measurements of LVEF, LVFS, LVESD and LVEDD. (g) Quantification of the early mitral diastolic wave/late mitral diastolic wave ratio (E/A ratio). Data are means and SEM $(n=12) . * p<0.05$ vs NTg; ${ }^{\dagger} p<0.05 \mathrm{vs} \mathrm{Tg}-M s t 1 ;{ }^{\ddagger} p<0.05 \mathrm{vs}$ $\mathrm{DM}+\mathrm{NTg}$
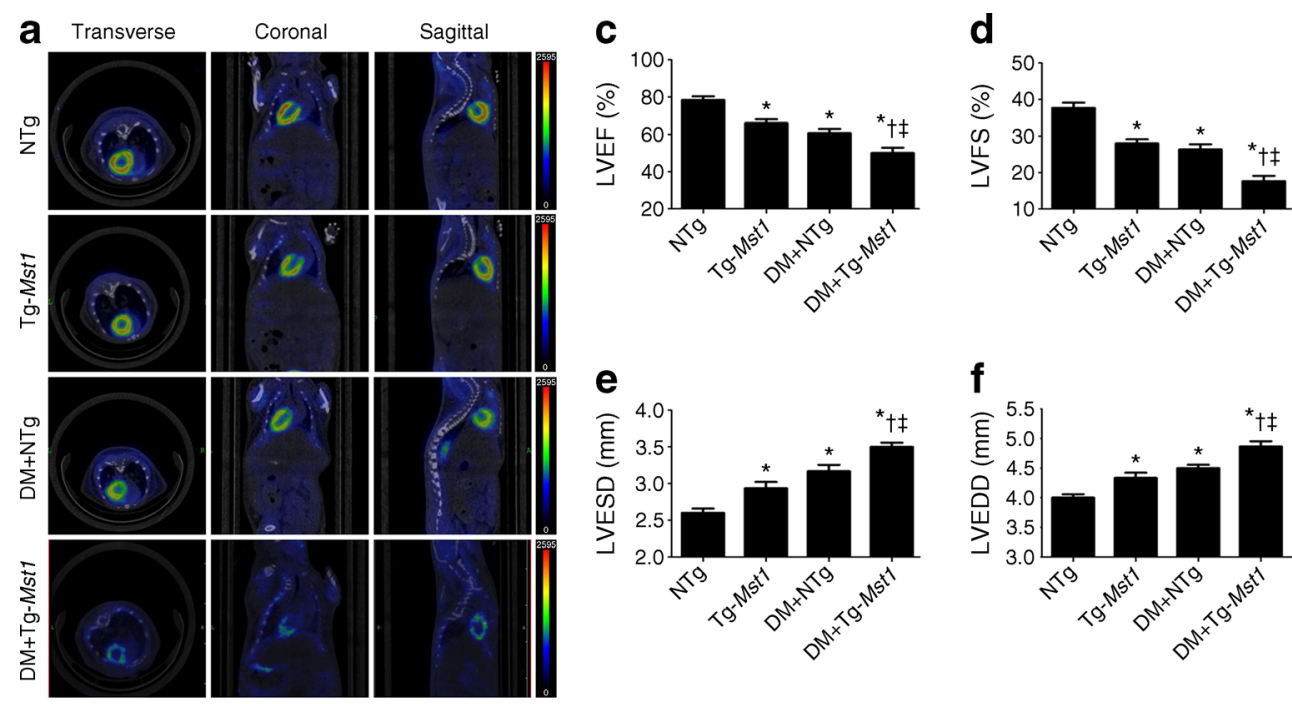

g

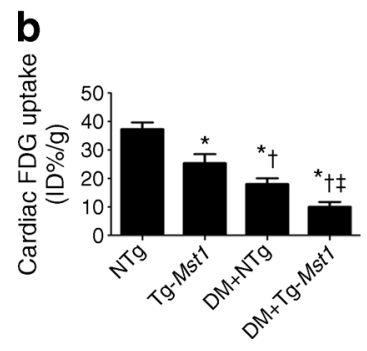

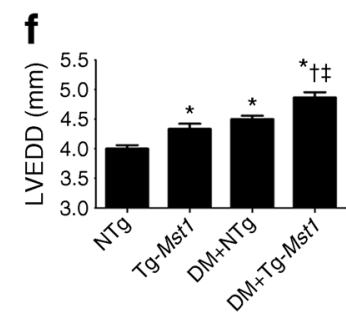

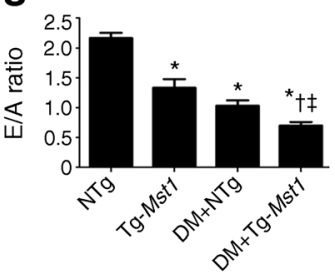


Mst1 overexpression aggravates cardiac dysfunction in diabetic mice Our data revealed that diabetes led to a defective ${ }^{18}$ F-FDG uptake in the heart, an effect that was significantly aggravated by Mst 1 overexpression (Fig. 5a, b). Representative M-mode echocardiograms are shown in ESM Fig. 6a. LVEF and LVFS were further decreased in the DM + Tg-Mst1 group compared with the DM + NTg group (Fig. 5c, d). Diabetic Mst1 transgenic mice exhibited increased LVESD and LVEDD as compared with the DM + NTg group (Fig. 5e, f). Representative mitral flow patterns from pulsed Doppler are shown in ESM Fig. 6b. The E/A ratio was further decreased in the DM + Tg-Mst1 group compared with the DM + NTg group (Fig. 5g). Baseline variables are shown in ESM Table 2. IPGTT results showed that the diabetic model was constructed successfully (ESM Fig. 7). There were significantly fewer autophagosomes in the Tg-Mst1 group compared with the NTg group regardless of diabetes state (ESM Fig. 8a). AdMst 1 transfection significantly decreased the number autophagosomes as compared with the control group under normal and high-glucose conditions (ESM Fig. 8b).

Mst1 overexpression inhibits autophagy and increases accumulation of protein aggresomes and p62 in cardiomyocytes There was a significant increase in p-MST1, MST1 and p-
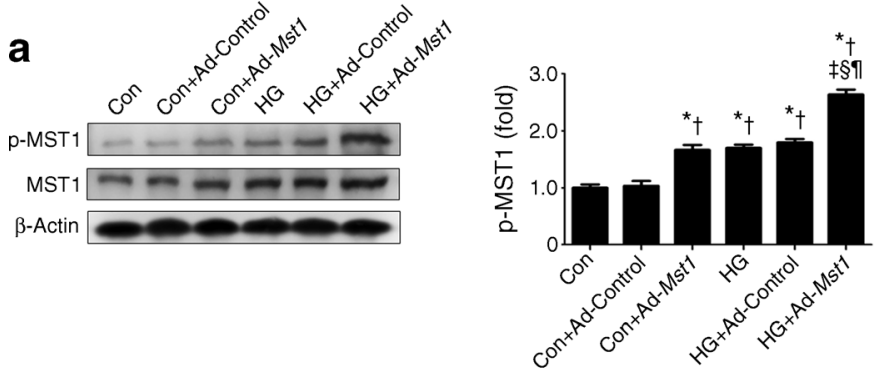
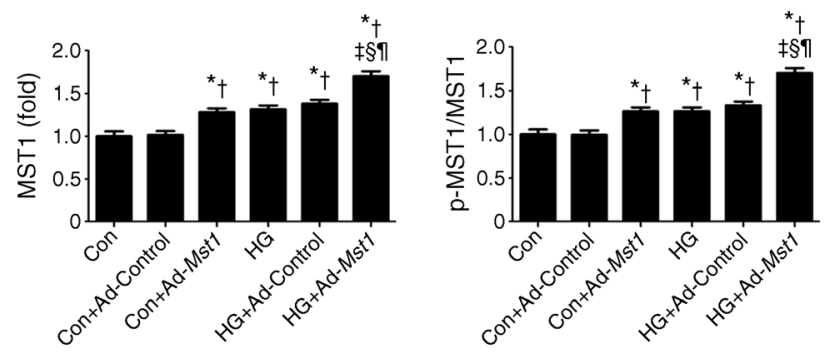

b

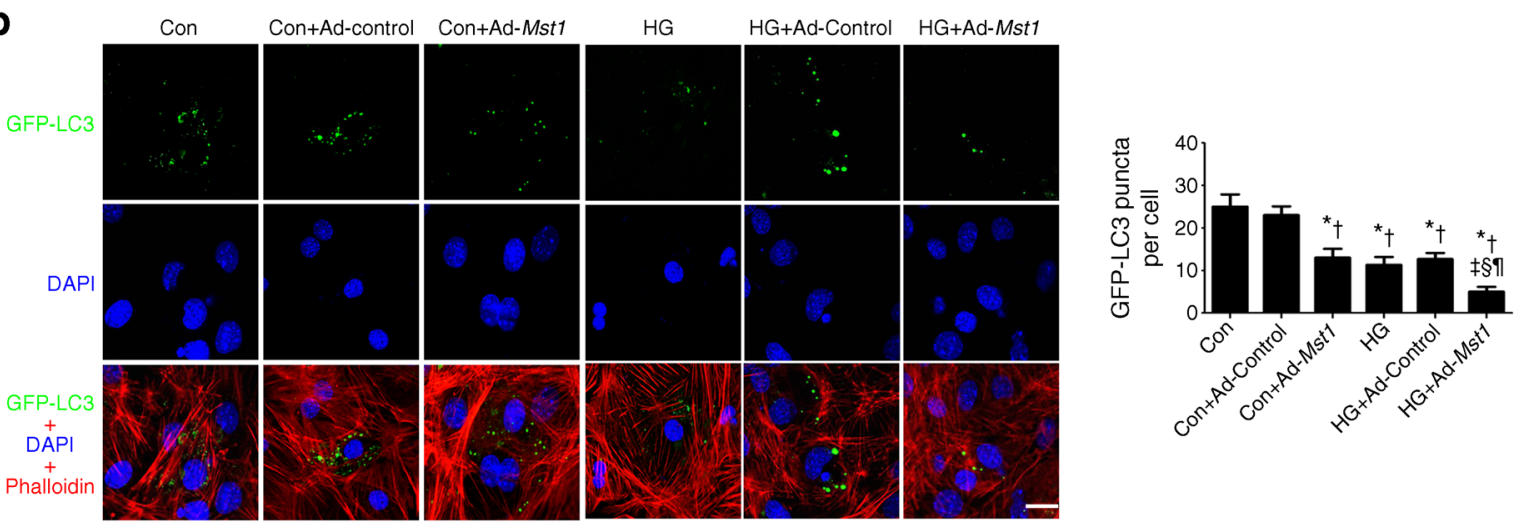

C
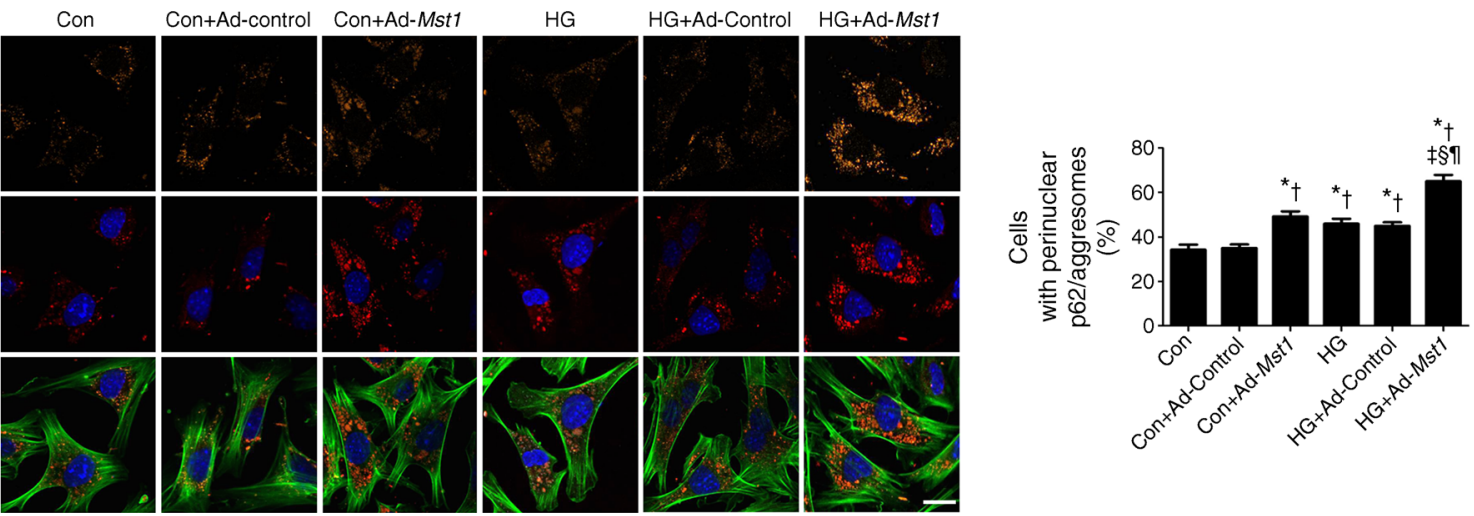

Fig. 6 Mst 1 overexpression inhibits autophagy and increases accumulation of protein aggresomes and p62 in cardiomyocytes. (a) Representative blots and analysis of p-MST1 and MST1 expression. (b) Representative images and quantitative analysis of GFP-LC3 puncta. (c) Representative images showing immunofluorescence staining for p62 (orange), DAPI (blue) and ProteoStat aggresome detection reagent (red) in cultured

cardiomyocytes. The number of cardiomyocytes with perinuclear colocalisation of p62 and aggresomes (p62/aggresomes), indicated by yellow colour in the merged images, was counted ( $n=50$ in each group). ${ }^{*} p<0.05$ vs Con; ${ }^{\dagger} p<0.05$ vs Con + Ad-Control; ${ }^{\star} p<0.05$ vs Con + AdMst $1 ;{ }^{\S} p<0.05$ vs HG; ${ }^{\text {II }} p<0.05$ vs HG + Ad-Control 

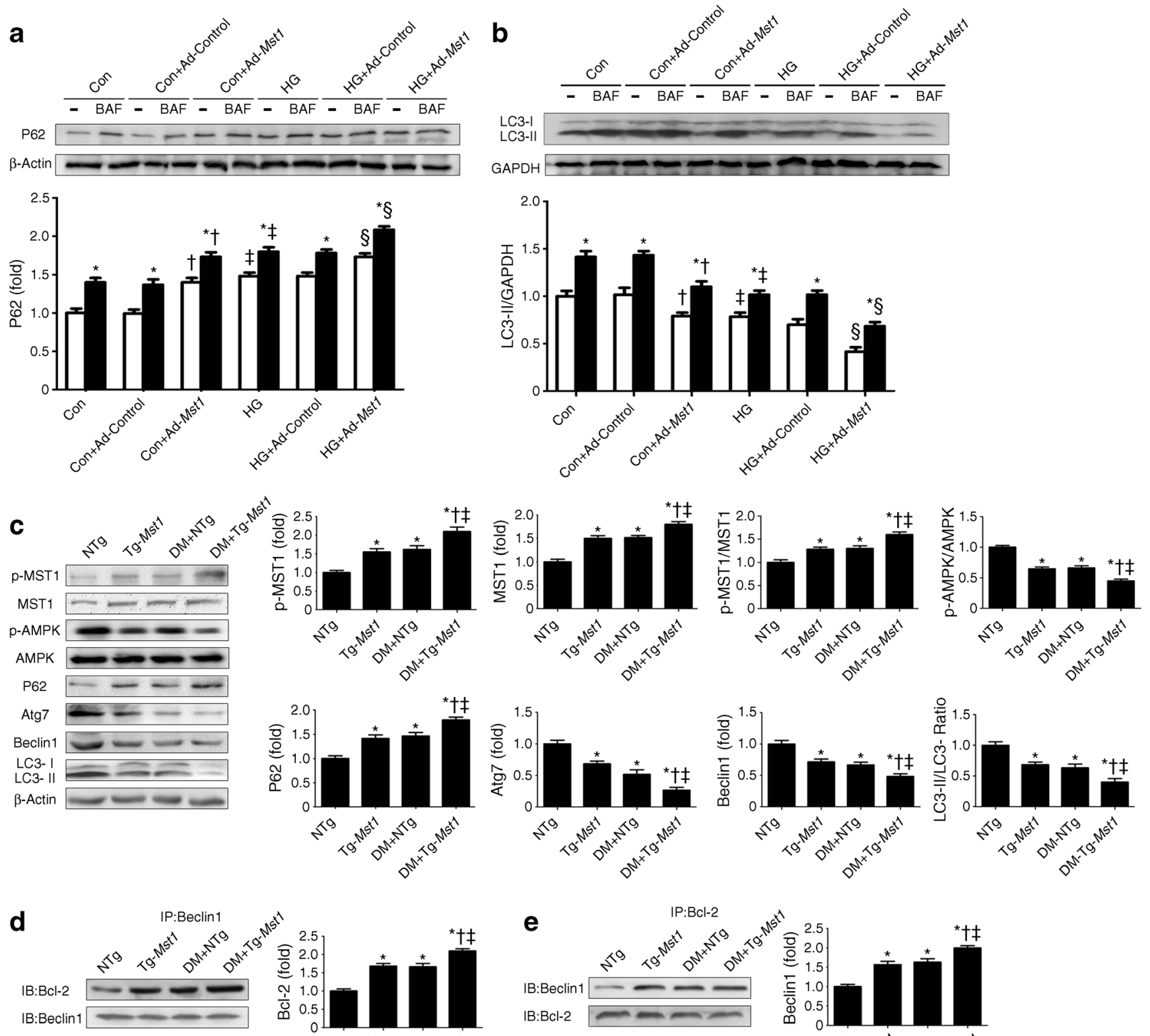
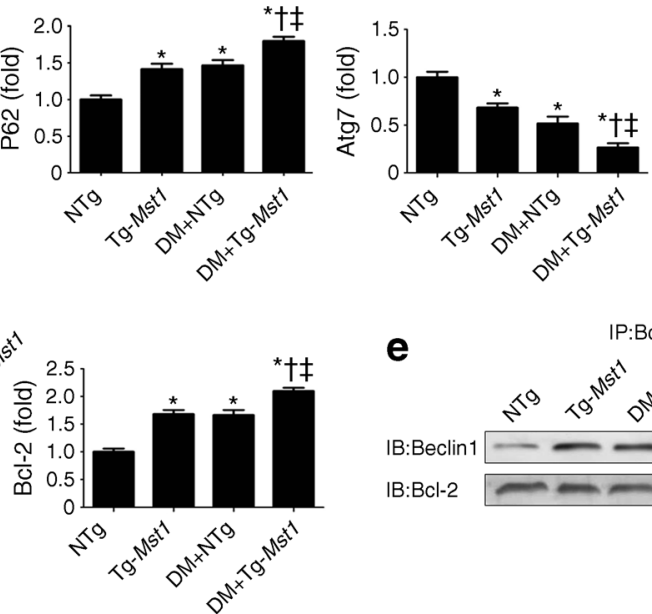

e
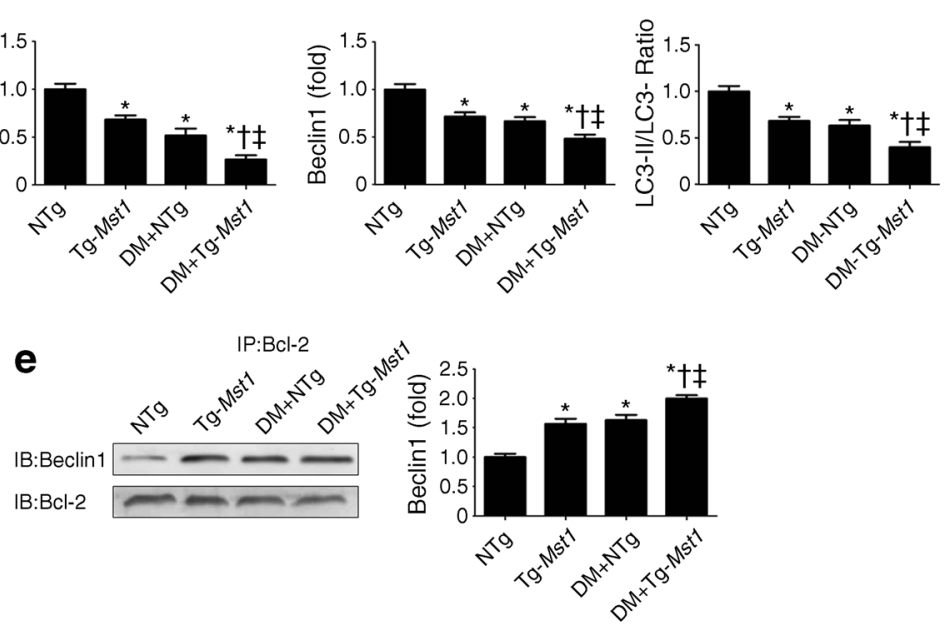

analyses of p-MST1, MST1, p-AMPK, AMPK, p62, Atg7, Beclin1 and LC3-II/LC3-I. (d, e) Beclin1 or Bcl-2 was immunoprecipitated (IP) from cell lysates and $\mathrm{Bcl}-2$ or Beclin1 in the immunoprecipitates was detected by western blot (IB). Data are means and SEM $(n=12) . * p<0.05$ vs NTg; ${ }^{\dagger} p<0.05$ vs Tg-Mst $1 ;{ }^{\star} p<0.05$ vs DM + NTg. Scale bars, $20 \mu \mathrm{m}$; BAF, bafilomycin A; Con, control; HG, high glucose

Mst1 overexpression inhibits autophagy by interaction of Beclin1 and Bcl-2 Ad-Mst1 transfection inhibited autophagic flux, as demonstrated by decreased LC3-II/GAPDH ratio and increased p62 expression in the presence of bafilomycin A1 (Fig. 7a, b). Consistent with this, western blot analysis revealed a reduction in the ratio of LC3-II/LC3-I and accumulation of $\mathrm{p} 62$ in the Tg-Mst1 mouse hearts compared with nontransgenic hearts regardless of diabetic state (Fig. 7c). In normal- and high-glucose conditions (Fig. 6c). 
a

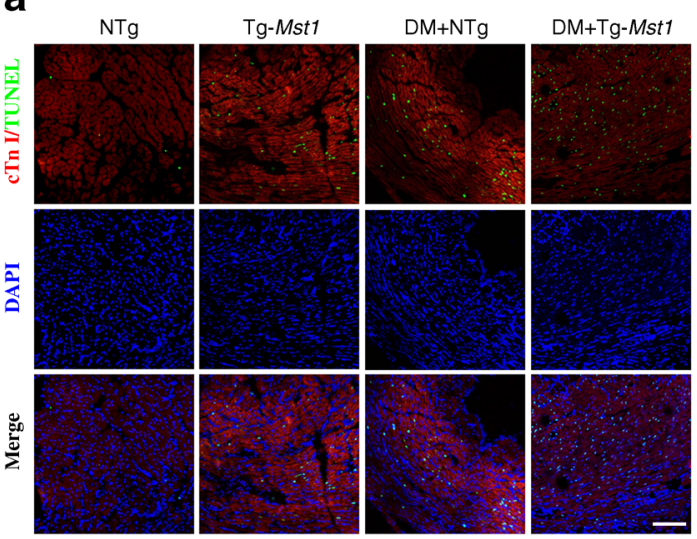

C
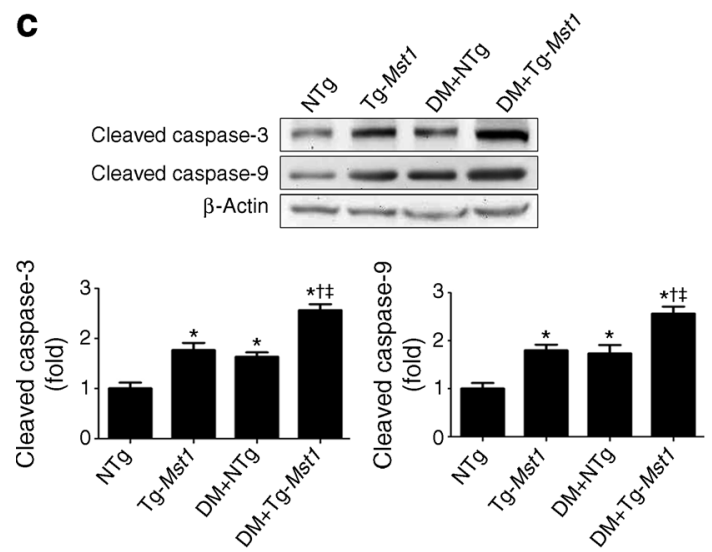

e
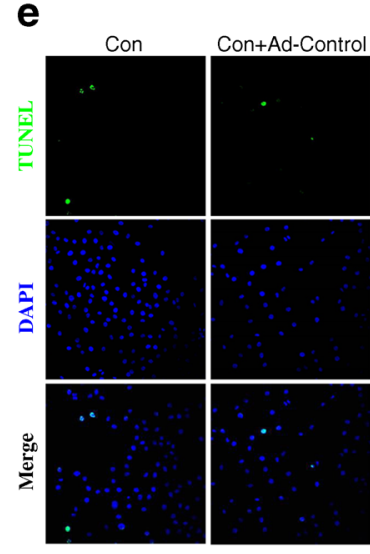

g

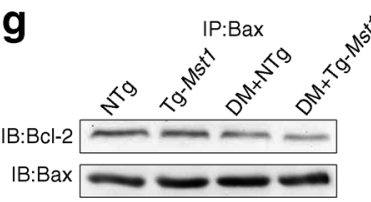

\section{b}

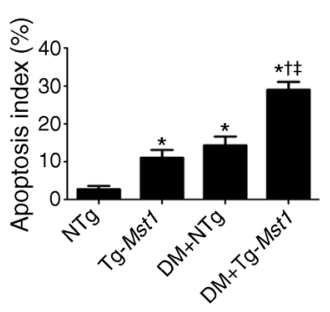

d

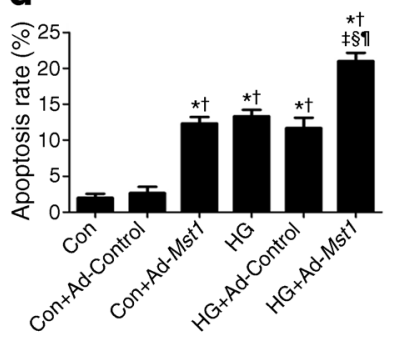

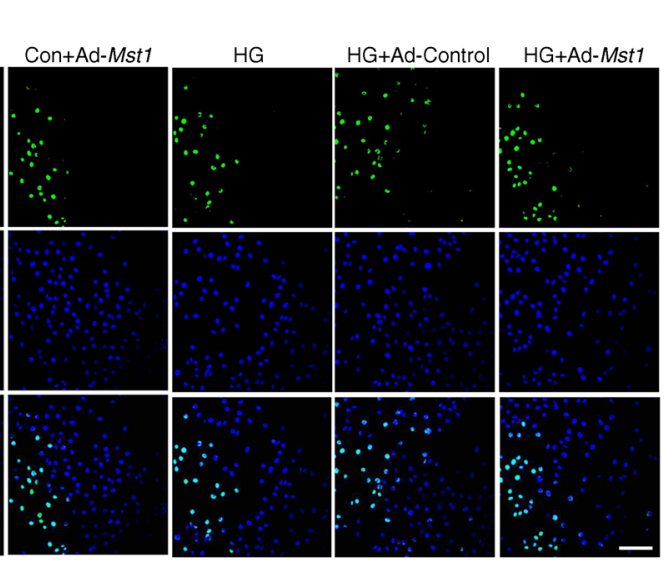
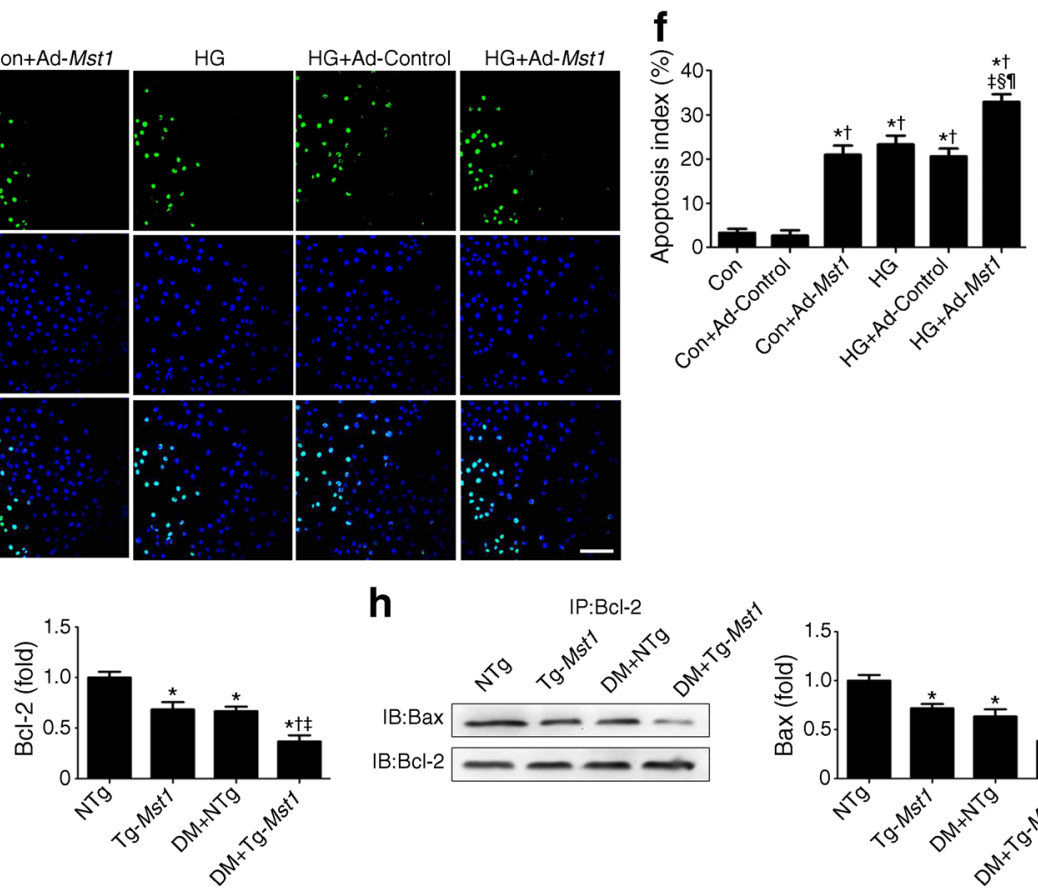

h

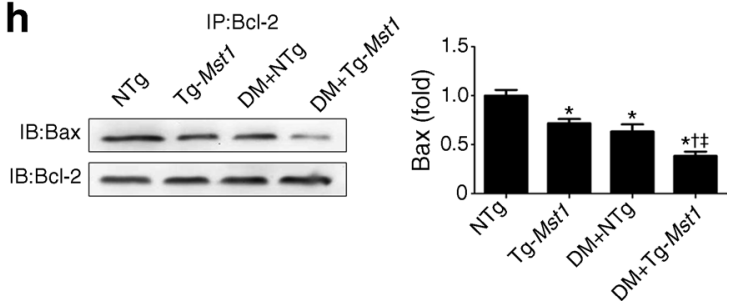

addition, the p-AMPK/AMPK ratio was further decreased in the $\mathrm{DM}+\mathrm{Tg}-M s t 1$ group as compared with the DM group (Fig. 7c). Co-immunoprecipitation assays revealed more binding of Bcl-2 to Beclin1 in the Tg-Mst 1 group than in the non- transgenic group regardless of diabetes status (Fig. 7d, e). These results further demonstrated that MST1 inhibition of autophagy is mediated by regulation of the association between Bcl-2 and Beclin1. Treatment with the AMPK activator 
Fig. 8 Mst 1 overexpression regulates the association of $\mathrm{Bax}$ and $\mathrm{Bcl}-2$ and increases apoptotic cells. (a, b) Representative immunofluorescent images of staining with TUNEL (green), DAPI (blue) and Troponin T antibody (cTn I; red) and quantitative analyses of apoptotic cells. Apoptotic index is expressed as the percentage of TUNEL-positive myocytes (in green) over total nuclei determined by DAPI staining ( $n=50$ fields per group). (c) Representative gel blots showing expression of cleaved caspase-3, cleaved caspase- 9 and $\beta$-actin (loading control) proteins. ${ }^{*} p<0.05$ vs NTg; ${ }^{\dagger} p<0.05$ vs Tg-Mst $1 ;{ }^{\ddagger} p<0.05$ vs $\mathrm{DM}+\mathrm{NTg}$. (d) Apoptosis rate = (late apoptotic cells + early apoptotic cells)/total cells $\times 100$. (e) Representative images of TUNEL-stained primary neonatal cardiomyocytes. (f) Apoptotic index expressed as the percentage of TUNEL-positive myocytes (in green) over total nuclei determined by DAPI staining ( $n=50$ fields per group). (g, h) Bax or $\mathrm{Bcl}-2$ was immunoprecipitated (IP) from cell lysates and $\mathrm{Bcl}-2$ or Bax in the immunoprecipitates was detected by western blot (IB). Data are means and SEM $(n=12)$. $* p<0.05$ vs Con; ${ }^{\dagger} p<0.05$ vs Con + AdControl; ${ }^{\ddagger} p<0.05$ vs Con + Ad-Mst $1 ;{ }^{\S} p<0.05$ vs HG; ${ }^{9} p<0.05$ vs HG + Ad-Control. Con, control; HG, high glucose

metformin significantly increased LVEF as well as LVFS and depressed LVESD and LVEDD in diabetic mice; consistently, the effects of Mst 1 overexpression were reversed partially by metformin administration in diabetic mice (ESM Fig. 9a-d).

\section{Mst1 overexpression induces cardiomyocyte apoptosis by} dissociation of Bax and Bcl-2 The percentage of TUNELpositive cardiomyocytes was significantly increased in the $\mathrm{DM}+\mathrm{Tg}-M$ st1 group when compared with the DM + NTg group (Fig. 8a, b). Furthermore, protein levels of cleaved caspase-3 and cleaved caspase- 9 were increased in diabetic hearts, with a more pronounced rise in Mst1-overexpressing transgenic mice (Fig. 8c). Flow cytometry and representative TUNEL images also demonstrated that Mst1 overexpression resulted in an increase in the ratio of apoptotic cardiomyocytes cultured in high-glucose medium (Fig. 8d-f). Compared with the $\mathrm{DM}+\mathrm{NTg}$ group, $M s t 1$ overexpression in diabetic mice significantly interrupted the binding between Bax and Bcl-2 as assessed using the co-immunoprecipitation assay (Fig. 8g, h). Flow cytometry analysis showed that Ad-Mst1 transfection significantly increased the apoptosis rate of cardiomyocytes as compared with the control group under high-glucose conditions (ESM Fig. 10).

\section{Discussion}

Diabetes mellitus is associated with significantly higher cardiovascular morbidity and mortality when compared with normoglycaemia. Heart failure in diabetic individuals has an alarmingly high prevalence, incidence and mortality, yet the underlying pathogenesis of DCM remains unclear [3-5, 16]. The major findings from our current study revealed decreased myocardial glucose uptake and impaired cardiac diastolic and systolic function in diabetic mice. Interestingly, Mst1 knockout improved myocardial glucose uptake and cardiac function in experimental DCM. Furthermore, Mst 1 overexpression aggravated the development of DCM. These data indicated that an MST1 inhibition-mediated physiological process may constitute a protective mechanism against DCM (Fig. 9).

Autophagy may be either protective or detrimental depending on the extent of autophagy and on cell type and cellular environment. In the heart, constitutive autophagy is a homeostatic mechanism involved in the maintenance of cardiac structure and function $[6,17]$. Suppression of autophagy to below the physiological level may promote heart failure with enhanced protein aggregation, suggesting a permissive role for autophagy in the maintenance of cardiac function [18].
Fig. 9 Schematic diagram depicting possible mechanisms involved in the protective effect of Mst1 knockout in diabetic cardiomyopathy

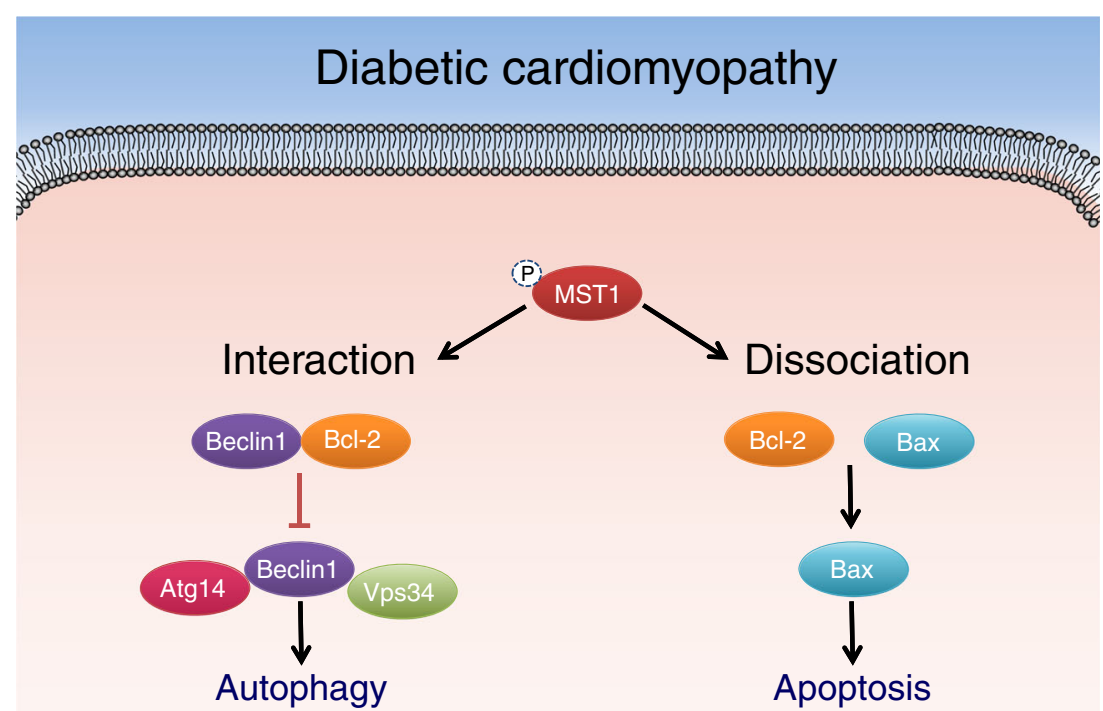


In our hands, diabetic Mst 1 transgenic mice overexpressing Mst 1 exhibited decreased LC3 levels and enhanced protein aggregation. In contrast, typical autophagosomes were observed in diabetic Mst1-knockout mice with increased LC3 expression and alleviated protein aggregation. Most notably, the protective effects of Mst 1 knockout in diabetic mice were reversed by administration of 3-MA, an inhibitor of autophagy. These observations provide some evidence to support the notion that MST1-suppressed cardiomyocyte autophagy may represent a critical event in the development of DCM.

Beclin1 is a Bcl-2 homology 3 (BH3) domain-only protein that plays an important part in autophagy initiation [19]. Accumulating evidence points to the existence of an interaction between Bcl-2 and Beclin1. The binding of Bcl-2 to Beclin1 inhibits autophagy via sequestration of Beclin1 away from class III phosphoinositide 3-kinase (PI3K) [20-22]. AMPK activation also results in the disruption of the association between Beclin1 and Bcl-2, allowing the free Beclin1 to bind to class III PI3K to form the kinase complex, which is essential for the induction of autophagy $[8,23]$. In the present study, the interaction between Beclin1 and Bcl-2 in hearts from diabetic mice was also investigated. Co-immunoprecipitation assays showed that Mstl overexpression promoted Beclin1 binding to Bcl-2. Conversely, Mstl knockout disengaged the Beclin1-Bcl-2 interplay, thus promoting autophagy and subsequent elimination of protein aggregates. Interestingly, we observed an increased p-AMPK/AMPK ratio in the Mst1-knockout mouse group as compared with the DM group. AMPK activator (metformin) attenuated Mst 1 overexpression-induced cardiac dysfunction in diabetic mice. These data indicate that Mst 1 overexpression inhibits AMPK phosphorylation and positively engages the interplay between Beclin1 and Bcl-2, thus leading to the inhibition of autophagy.

The interplay between autophagy and apoptosis is pivotal in the pathogenesis of DCM [8]. Several studies have demonstrated that increased autophagy promotes cell survival under conditions of nutrient deprivation or growth factor withdrawal through inhibition of apoptosis [24, 25]. MST1 is a pro-apoptotic kinase that also regulates autophagy. The ability of MST1 to regulate both autophagy and apoptosis may contribute to the development of DCM. In the current study, Mst1 overexpression increased cardiomyocyte apoptosis both in vivo and in vitro. Bax is also a BH3 domain-containing protein that can bind to Bcl-2. Attenuation of the interaction between Bcl-2 and Bax enhances apoptosis by allowing Bax to take an active conformation. We then examined whether MST1 disturbs the interaction between $\mathrm{Bcl}-2$ and $\mathrm{Bax}$ in addition to promoting the interaction between Beclin1 and Bcl-2. As expected, Mst1 knockout enhanced the interaction between Bcl-2 and Bax in diabetic mice. Thus, an MST1-induced dissociation of Bcl-2 from Bax, which enhances apoptosis, may also contribute to the development of DCM.

In summary, we demonstrate here that MST1, a proapoptotic signalling kinase, may facilitate the progression of DCM by inhibiting autophagy. MST1 induces interdependent modulation of Beclin1, Bcl-2 and Bax, thereby regulating autophagy and apoptosis simultaneously (Fig. 7). Our results provide new insight into the regulation of autophagy and apoptosis in the development of DCM. MST1 may represent a novel target for the treatment of heart failure in diabetic individuals.

Funding This work was supported by the National Natural Science Foundation of China (grant no. 81570318, 81570361, 81300149 and 81270263), Shaanxi Social Development and Scientific Problem Tackling Program (2015SF097, 2015KW-036).

Duality of interest The authors declare that there is no duality of interest associated with this manuscript.

Contribution statement DS, HW, MZ, LZ, JH and JL designed the experiments, analysed and interpreted the data and drafted the manuscript. DS, MZ, TW, YD, WM, JF, LS, HJ, CL and RZ were involved in the data acquisition and analysis. All authors revised the manuscript critically for important intellectual content and approved the final version to be published. DS is responsible for the integrity of the work as a whole.

\section{References}

1. Wild S, Roglic G, Green A, Sicree R, King H (2004) Global prevalence of diabetes: estimates for the year 2000 and projections for 2030. Diabetes Care 27:1047-1053

2. Kobayashi S, Liang Q (2015) Autophagy and mitophagy in diabetic cardiomyopathy. Biochim Biophys Acta 1852:252-261

3. Picano E (2003) Diabetic cardiomyopathy. the importance of being earliest. J Am Coll Cardiol 42:454-457

4. Avogaro A, Vigili de Kreutzenberg S, Negut C, Tiengo A, Scognamiglio R (2004) Diabetic cardiomyopathy: a metabolic perspective. J Am Coll Cardiol 93:13A-16A

5. Boudina S, Abel ED (2007) Diabetic cardiomyopathy revisited. Circulation 115:3213-3223

6. Maejima Y, Kyoi S, Zhai P et al (2013) Mst1 inhibits autophagy by promoting the interaction between Beclin1 and Bcl-2. Nat Med 19: $1478-1488$

7. Xie Z, Lau K, Eby B et al (2011) Improvement of cardiac functions by chronic metformin treatment is associated with enhanced cardiac autophagy in diabetic OVE26 mice. Diabetes 60:1770-1778

8. He C, Zhu H, Li H, Zou MH, Xie Z (2013) Dissociation of Bcl-2Beclin 1 complex by activated AMPK enhances cardiac autophagy and protects against cardiomyocyte apoptosis in diabetes. Diabetes 62:1270-1281

9. Guo Y, Yu W, Sun D et al (2015) A novel protective mechanism for mitochondrial aldehyde dehydrogenase (ALDH2) in type $i$ diabetes-induced cardiac dysfunction: role of AMPK-regulated autophagy. Biochim Biophys Acta 1852:319-331

10. Del Re DP, Matsuda T, Zhai P et al (2014) Mst1 promotes cardiac myocyte apoptosis through phosphorylation and inhibition of BclxL. Mol Cell 54:639-650

11. Odashima M, Usui S, Takagi H et al (2007) Inhibition of endogenous Mst1 prevents apoptosis and cardiac dysfunction without affecting cardiac hypertrophy after myocardial infarction. Circ Res 100:1344-1352

12. Yamamoto S, Yang G, Zablocki D et al (2003) Activation of Mst1 causes dilated cardiomyopathy by stimulating apoptosis without 
compensatory ventricular myocyte hypertrophy. J Clin Invest 111: $1463-1474$

13. Wang D, Luo P, Wang Y et al (2013) Glucagon-like peptide-1 protects against cardiac microvascular injury in diabetes via a cAMP/PKA/Rho-dependent mechanism. Diabetes 62:1697-1708

14. Hu J, Man W, Shen M et al (2015) Luteolin alleviates postinfarction cardiac dysfunction by up-regulating autophagy through Mst1 inhibition. J Cell Mol Med 20:147-156

15. Zhang M, Wang C, Hu J et al (2015) Notch3/Akt signaling contributes to OSM-induced protection against cardiac ischemia/ reperfusion injury. Apoptosis 20:1150-1163

16. Bertoni AG, Hundley WG, Massing MW, Bonds DE, Burke GL, Goff DC Jr (2004) Heart failure prevalence, incidence, and mortality in the elderly with diabetes. Diabetes Care 27:699-703

17. Martinet W, Knaapen MW, Kockx MM, De Meyer GR (2007) Autophagy in cardiovascular disease. Trends Mol Med 13:482-491

18. Nakai A, Yamaguchi O, Takeda T et al (2007) The role of autophagy in cardiomyocytes in the basal state and in response to hemodynamic stress. Nat Med 13:619-624
19. Liang XH, Kleeman LK, Jiang HH et al (1998) Protection against fatal Sindbis virus encephalitis by beclin, a novel Bcl-2-interacting protein. J Virol 72:8586-8596

20. Pattingre S, Tassa A, Qu X et al (2005) Bcl-2 antiapoptotic proteins inhibit Beclin 1-dependent autophagy. Cell 122:927-939

21. Liang XH, Jackson S, Seaman M et al (1999) Induction of autophagy and inhibition of tumorigenesis by beclin 1 . Nature 402:672-676

22. Levine B, Sinha S, Kroemer G (2008) Bcl-2 family members: dual regulators of apoptosis and autophagy. Autophagy 4:600-606

23. Maiuri MC, Le Toumelin G, Criollo A et al (2007) Functional and physical interaction between $\mathrm{Bcl}-\mathrm{X}(\mathrm{L})$ and a $\mathrm{BH} 3$-like domain in Beclin-1. EMBO J 26:2527-2539

24. Boya P, Gonzalez-Polo RA, Casares N et al (2005) Inhibition of macroautophagy triggers apoptosis. Mol Cell Biol 25: $1025-1040$

25. Lum JJ, Bauer DE, Kong M et al (2005) Growth factor regulation of autophagy and cell survival in the absence of apoptosis. Cell $120: 237-248$ 